\title{
Pensamiento crítico en ESO y Bachillerato: perspectiva de docentes y estudiantes de un IES en una provincia del norte de España
}

\author{
Critical thinking in ESO and Bachillerato: perspective of teachers and students in an \\ IES in a northern Spanish province
}

\author{
Diego Agudo Saiz (iD \\ e-mail: diegoagudosaiz@gmail.com \\ Universidad de Cantabria, España \\ Irina Salcines Talledo \\ e-mail: irina.salcines@unican.es \\ Universidad de Cantabria, España
}

Natalia González Fernández

e-mail: natalia.gonzalez@unican.es

Universidad de Cantabria, España

\begin{abstract}
Resumen
En los últimos años, se ha prestado un interés significativo a la promoción del pensamiento crítico de los estudiantes de ESO y Bachillerato en España. Sin embargo, la atención se ha centrado en contrastar la validez de propuestas didácticas diseñadas a tal efecto, dejando de lado el análisis de las perspectivas de los protagonistas del proceso educativo: los estudiantes y el profesorado. Mediante metodología mixta, esta investigación presenta un estudio de caso en el que se analiza el punto de vista de los docentes y discentes de un Instituto de Educación Secundaria de una provincia del norte de España en relación al desarrollo del pensamiento crítico del alumnado en las etapas mencionadas. Participaron en el estudio 25 docentes y 43 estudiantes, utilizándose cuestionarios elaborados ad hoc como instrumentos de recogida de información. Tanto docentes como estudiantes señalan el pensamiento crítico como un aspecto clave a desarrollar en ESO y Bachillerato, y perciben la utilidad de las actividades para fomentar el pensamiento crítico del alumnado, estando también de acuerdo en que su promoción se traduce en la obtención de mejores calificaciones. Ambos colectivos detectan carencias en la promoción del pensamiento crítico del alumnado, identificándose los principales puntos débiles en la conceptualización del pensamiento crítico y su evaluación.

Palabras clave: pensamiento; educación; estudiante; docente; investigación pedagógica.
\end{abstract}

\section{Abstract}

In recent years, the development of critical thinking of ESO and Bachillerato Spanish students has been paid a significant amount of attention. Nevertheless, the focus research topic has been trying to contrast the validity of didactic proposals designed with this purpose, leaving aside the analysis of the perspectives of the process protagonists: teachers and students. With a mixed methodology, this research presents a case study in which the point of view of the teachers and students at a high school located in the north of Spain, regarding the promotion of critical thinking of the ESO and Bachillerato students', is analyzed. 25 teachers and 43 students participated in the study, and ad hoc designed questionnaires were used as information gathering instruments. Both teachers and students pointed out that critical thinking was a key aspect to be developed in ESO and Bachillerato, and they perceived the utility of the activities designed to promote critical thinking amongst students. They also affirmed that fostering students' critical thinking would result in better grades. Both collectives signaled that the promotion of the students critical thinking was lackluster, identifying critical thinking conceptualization and evaluation as the main weak spots.

Keywords: thinking; education; students; teachers; educational research.

Recibido / Received: 14-02-2020

Aceptado / Accepted: 01-10-2020

Publicado en línea / Published online: 22-12-2020

Cómo referenciar este artículo / How to reference this article:

Agudo-Saiz, D., Salcines-Talledo, I., \& González-Fernández, N. (2021). Pensamiento crítico en ESO y Bachillerato: perspectiva de docentes y estudiantes de un IES en una provincia del norte de España. Tendencias Pedagógicas, 37, pp. 121-133. doi: 10.15366/tp2021.37.010 


\section{Introducción}

La promoción del pensamiento crítico del alumnado se encuentra entre los objetivos establecidos en la legislación que regula las etapas de ESO y Bachillerato en España (LOE, 2006; LOMCE, 2013). Recientemente, se han desarrollado diversas investigaciones en el ámbito nacional con el objetivo de analizar la efectividad de diversas propuestas didácticas para potenciar el pensamiento crítico del alumnado de dichas etapas (Albertos \& De la Herrán, 2018; Martínez, Ballester \& Ibarra, 2018; Úbeda-Colomer \& Molina, 2016). Sin embargo, los esfuerzos se han concentrado en la validación de las propuestas pedagógicas, dejando de lado el análisis de las perspectivas y actitudes frente al desarrollo del pensamiento crítico del alumnado de los principales actores involucrados: docentes y discentes.

Este estudio persigue analizar la perspectiva de profesorado y alumnado en relación a la promoción del pensamiento crítico de estos últimos, en las etapas de ESO y Bachillerato en el contexto de un Instituto de Educación Secundaria (IES), ubicado en la provincia del norte de España, durante el curso académico 2018-2019. Simultáneamente, se investiga sobre las concepciones que los integrantes de ambos colectivos poseen sobre el pensamiento crítico, así como las prácticas, estrategias y/o metodologías que se emplean en el centro educativo en la actualidad con el propósito de fomentar dicho pensamiento en alumnado.

\section{Revisión de la literatura}

En el análisis documental de la literatura especializada, no se ha encontrado ninguna publicación que estudie la perspectiva de los docentes o discentes en relación al desarrollo del pensamiento crítico de los últimos en ninguna etapa del sistema educativo español, sin embargo, se extendió la búsqueda a nivel internacional, encontrándose las evidencias en relación al fenómeno de estudio que se desarrollan seguidamente.

La promoción del pensamiento crítico del alumnado genera importantes beneficios ya que este hecho mejora sus capacidades de aprendizaje (Chee \& Kin, 2009; Innabi \& El Sheikh, 2006). Investigaciones realizadas en Estados Unidos y Malasia indican que, el profesorado universitario de dichos contextos educativos, considera necesario desarrollar el pensamiento crítico de los estudiantes y, además, cree firmemente incluir este aspecto en su práctica docente (Chee \& Kin, 2009; Kowalczyk, Hackworth \& Case-Smith, 2012). En contraposición, en el contexto iraní, los docentes de las citadas etapas educativas no consideran el pensamiento crítico como un aspecto necesario en la formación de sus estudiantes (Aliakbari \& Sadeghdaghighi, 2013), por lo que el contexto sociocultural parece tener impacto en el fenómeno de estudio.

La perspectiva de los estudiantes es, en ocasiones, contraria a la de los docentes, ya que, desde su punto de vista, no se presta suficiente atención al desarrollo de su pensamiento crítico en su formación académica, como muestra la investigación llevada a cabo por Tapper (2004) en el contexto universitario australiano. En lo que respecta a los futuros docentes de Educación Secundaria, el estudio efectuado por Allamnakhrah (2013) en Arabia Saudí indica, de la misma manera, que el pensamiento crítico no está en las bases de su formación como docentes, señalando al profesorado universitario como el mayor responsable tras este hecho, argumentando que emplean metodologías tradicionales basadas en la transmisión de conocimientos, y no en el protagonismo de los estudiantes en su proceso de aprendizaje.

En cuanto a la metodología empleada por los docentes para promover el pensamiento crítico de su alumnado, el profesorado universitario europeo señala que su preferencia es el enfoque de inmersión, es decir, integrando el pensamiento crítico en su enseñanza diaria, pero sin abordarlo de manera específica (Dumitru, 2018). Un hecho similar se apreció en la investigación de Tapper (2004), al observarse que es frecuente que el pensamiento crítico de los estudiantes se desarrolle gracias a la práctica, sin necesidad de que se incida explícitamente sobre su promoción. Entre las formas de intervención específicas destacadas por los docentes universitarios europeos se encuentran el diálogo y el análisis de situaciones reales (Dumitru, 2018).

Constituye un aspecto importante que, tanto en Educación Secundaria como Universitaria, el profesorado es tendente a cometer errores en la conceptualización del pensamiento crítico, mostrando 
además carencias en el conocimiento de herramientas para promoverlo en los estudiantes (Alazzi, 2008; Aliakbari \& Sadeghdaghighi, 2013; Chee \& Kin, 2009; Innabi \& El Sheikh, 2006).

Los docentes identifican barreras a la hora de promover el pensamiento crítico del alumnado. En determinados contextos educativos, dicha promoción se puede ver obstaculizada por aspectos culturales (Alazzi, 2008; Allamnakhrah, 2013). Por otra parte, Chee \& Kin (2009) señalan que algunos estudiantes pueden sentirse incómodos cuando se realizan actividades para desarrollar actividades para desarrollar su pensamiento crítico. De la misma forma, otros pueden ser contrarios a realizar este tipo de actividades por el trabajo e implicación que requieren por su parte (Allamnakhrah, 2013). Otra barrera puede ser temporal, causada por un gran volumen de contenidos en el currículo, lo que dificulta seriamente que se pueda promover el pensamiento crítico de los estudiantes, ya que es habitual priorizar la transmisión de contenidos frente al desarrollo de esta competencia (Chee \& Kin, 2009; Kowalczyk, Hackworth \& Case-Smith, 2012).

Los obstáculos en la promoción del pensamiento crítico de los estudiantes son sensibles a las circunstancias del contexto educativo. Por ejemplo, los docentes universitarios estadounidenses destacan el reducido apoyo institucional y la escasa financiación para ser provistos de materiales para enseñar pensamiento crítico (Kowalczyk, Hackworth \& Case-Smith, 2012). Por el contrario, en Irán, Aliakbari \& Sadeghdaghighi (2013) indican que ni el tiempo ni las barreras institucionales constituyen obstáculos, pero señalan la dificultad y el trabajo por su parte que implica aplicar prácticas, estrategias y metodologías para promover el pensamiento crítico del alumnado.

Se ha observado en diversos estudios que la edad, los años de experiencia docente y la aplicación de reformas educativas, son aspectos que no tienen impacto sobre la perspectiva del profesorado en relación a la promoción del pensamiento crítico de los estudiantes (Innabi \& El Sheikh, 2006; Kowalczyk, Hackworth \& Case-Smith, 2012).

Por último, los docentes señalan varios aspectos que son susceptibles de mejora. El profesorado universitario estadounidense, pese a mostrarse confiados a la hora de promover el pensamiento crítico del alumnado, indicaron desear tener un mayor desarrollo profesional en esta área (Kowalczyk, Hackworth \& Case-Smith, 2012). Por otra parte, en el contexto europeo, Dumitru (2018) señala que los docentes universitarios encuentran difícil promover el pensamiento crítico de los estudiantes si esto se realiza solo en una asignatura, abogando por incluirlo de manera integral en los planes de estudio. Además, indica que el aspecto más deficitario se encuentra en las fases de evaluación, ya que la mayoría de docentes admiten no incluir el pensamiento crítico en dichos estadios.

Puede concluirse de esta revisión que, el análisis de la perspectiva del alumnado y el profesorado frente a la promoción del pensamiento crítico de los estudiantes, es un tema que ha sido abordado por varias investigaciones a nivel internacional, centrándose los estudios en las etapas de Educación Secundaria y Universitaria. La promoción del pensamiento crítico en Educación Secundaria y Universitaria se encuentra íntimamente relacionada, al constituir el desarrollo de esta competencia un proceso continuo. Además, los estudiantes tienden a comparar la forma en que su pensamiento crítico se desarrolla en la Universidad, con su experiencia en Educación Secundaria (Tapper, 2004). Las publicaciones ofrecen datos sobre las perspectivas de los colectivos citados, aunque debe tenerse presente que es posible que, los puntos de vista respecto al fenómeno de estudio, difieran en función del contexto educativo.

\section{Método}

\subsection{Enfoque metodológico}

El método seleccionado para la investigación es el estudio de caso, considerándose óptimo para analizar un fenómeno de estudio en un contexto determinado. El método de estudio de caso es frecuentemente utilizado en investigación educativa (Cárdenas, Farias \& Méndez, 2017; Lima, Ávila \& López, 2015; Lozano \& Vieites, 2017).

El estudio de caso es generalmente considerado un método de corte cualitativo y diseñado de acuerdo a esta metodología. Sin embargo, algunos autores defienden la combinación de metodologías cuantitativas y cualitativas en los estudios de caso con el objetivo de obtener una visión más integral 
del fenómeno analizado (Yazan, 2015). Siguiendo esta última afirmación, la investigación opta por emplear metodología cuantitativa y cualitativa, por lo que adopta un enfoque mixto.

\subsection{Muestra}

La muestra estuvo formada la totalidad del profesorado del centro de un IES localizado en una provincia del norte de España (60 docentes), y por el alumnado de varios grupos de las asignaturas de Tecnología y Tecnología Industrial, pertenecientes a los niveles de $3^{\circ} \mathrm{ESO}, 4^{\circ} \mathrm{ESO}, 1^{\circ}$ Bachillerato y $2^{\circ}$ Bachillerato (45 estudiantes).

El muestreo realizado a la hora de seleccionar los grupos de estudiantes para el estudio fue de tipo accidental no probabilístico, siendo elegidos los grupos de estudiantes por motivos de accesibilidad por parte del investigador (Albert, 2007; Bisquerra, 2004). En la Figura 1 se esquematiza la distribución por género y colectivo de los individuos que conforman la muestra.

Figura 1.

Distribución por género y colectivo de los integrantes de la muestra

\section{$\underline{\text { N.o docentes por género }}$}

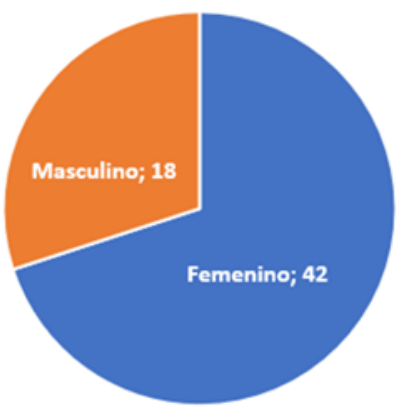

N. estudiantes por género

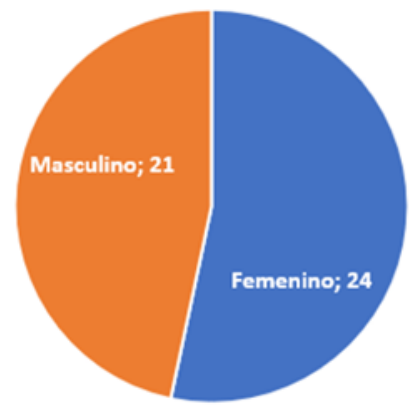

$\underline{\text { N.o estudiantes por curso }}$

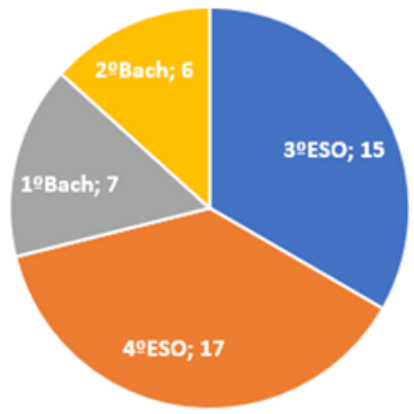

Fuente: elaboración propia

Al tratarse de una investigación centrada en un único centro educativo, es un aspecto a destacar que el tamaño de la muestra es reducido.

\subsection{Instrumentos de recogida de información}

Con el objetivo de recoger datos sobre la perspectiva docente en relación a la promoción del pensamiento crítico del alumnado en las etapas de ESO y Bachillerato se empleó un cuestionario elaborado ad hoc, denominado «Percepción docente sobre el desarrollo del pensamiento crítico del alumnado en ESO y Bachillerato». El cuestionario se divide en tres bloques, con preguntas sociodemográficas, cuestiones cerradas de valoración mediante escala tipo Likert de 4 niveles y preguntas abiertas de redacción libre (Anexo I). Las preguntas abiertas fueron seleccionadas y adaptadas de las cuestiones planteadas por Dumitru et al. (2018) para entrevistar a profesores sobre su posición en relación a la promoción del pensamiento crítico de sus estudiantes. El cuestionario para analizar la perspectiva docente respecto al pensamiento crítico, se estructura de acuerdo a lo mostrado en la Tabla 1.

Tabla 1.

Relación entre los parámetros del estudio y los contenidos del cuestionario docente sobre la promoción del pensamiento crítico del alumnado

\begin{tabular}{lll}
\hline Apartado & Descripción & Forma de recogida de información \\
\hline A & Datos sociodemográficos & 2 ítems cerrados (Ítems C1 y C2) \\
& & 1 pregunta abierta (Ítem C3) \\
B & Evaluación cuantitativa sobre pensamiento crí- & 10 ítems cerrados con valoración me- \\
& tico & diante escala tipo Likert (Ítems 1 a 10) \\
C & Evaluación cualitativa sobre pensamiento crítico & 2 preguntas abiertas de redacción libre \\
& & (Ítems 11 y 12) \\
\hline
\end{tabular}

Fuente: elaboración propia. 
Para la recogida de información sobre la perspectiva del alumnado se utilizó también un cuestionario elaborado ad hoc, siguiendo los mismos preceptos en su confección y estructura que los establecidos para el cuestionario destinado a los docentes.

Ambos cuestionarios fueron sometidos a un proceso de validación siguiendo el método Delphi, consistente en un proceso iterativo de interrogación a jueces expertos o panelistas con el objetivo de conocer sus opiniones respecto a la validez de las herramientas (Briceño \& Romero, 2012). Las cinco panelistas fueron seleccionadas bajo criterios de formación, trayectoria profesional y experiencia. Tras la elaboración de las herramientas iniciales, basadas en la revisión teórica, estas fueron remitidas a las panelistas para su revisión, empleando para dicha valoración la plantilla confeccionada por SalcinesTalledo \& González-Fernández (2015). Fueron necesarias dos iteraciones, reelaborando las herramientas de acuerdo a la realimentación proporcionada por el panel de expertos, y volviéndolas a someter al proceso de revisión en cada una de ellas, para alcanzar el consenso de las panelistas respecto a la validez de las herramientas y obtener las herramientas definitivas. Durante el proceso de revisión, surgió la división por bloques presente en el cuestionario definitivo y se solventaron problemas de relevancia y falta de univocidad en las preguntas.

Las variables del estudio, sobre las que los cuestionarios buscan proporcionar información, se presentan en la Tabla 2.

Tabla 2.

Tabla de variables

\begin{tabular}{lllll}
\hline Variable & Nombre & Descripción & Carácter & Tipo \\
\hline V1 & PC-Docente & $\begin{array}{l}\text { Grado en que los docentes consideran que el } \\
\text { pensamiento crítico del alumnado es promo- } \\
\text { vido en el centro }\end{array}$ & Dependiente & Escala \\
V2 & PC-Alumnado & $\begin{array}{l}\text { Grado en que el alumnado considera que su } \\
\text { pensamiento crítico es promovido en el centro }\end{array}$ & Dependiente & Escala \\
VS1 & Género & Género del participante & Independiente & Nominal \\
VS2 & Experiencia & Años de experiencia docente & Independiente & Nominal \\
VS3 & Asignaturas & Asignaturas impartidas por el docente & Independiente & Nominal \\
VS4 & Nivel & Nivel en que se encuentra el estudiante & Independiente & Nominal \\
\hline
\end{tabular}

Fuente: elaboración propia.

Las variables sociodemográficas (VS1, VS2, VS3 y VS4), se incluyen con el objetivo de no obviar factores con potencial influencia sobre los fenómenos estudiados.

\subsection{Trabajo de campo}

Los cuestionarios destinados a los docentes fueron digitalizados a través de la plataforma LimeSurvey y distribuidos por correo electrónico por la directora del centro. En el caso del alumnado, los cuestionarios fueron entregados en formato papel a los estudiantes en una de sus sesiones lectivas, quienes lo devolvieron cumplimentado en el momento. El tiempo requerido para la respuesta de ambos cuestionarios, se situó en torno a 10-15 minutos.

\subsection{Análisis de datos}

\subsubsection{Datos cuantitativos}

Teniendo en cuenta que los bloques cuantitativos de los cuestionarios han sido diseñados incluyendo ítems redactados desde perspectivas positivas y negativas, tratando de evitar tendencias en la respuesta, los datos adquiridos a través de los cuestionarios se codifican de acuerdo a lo indicado en la Tabla 3, siendo TD (Totalmente en Desacuerdo), D (Desacuerdo), A (De Acuerdo) y TA (Totalmente de Acuerdo). 
Tabla 3.

\begin{tabular}{|c|c|c|c|c|c|c|c|c|c|}
\hline \multicolumn{5}{|c|}{$\begin{array}{l}\text { Cuestionario percepción } \\
\text { PC docente }\end{array}$} & \multicolumn{5}{|c|}{$\begin{array}{l}\text { Cuestionario percepción } \\
\text { PC alumnado }\end{array}$} \\
\hline Ítem & TD & $\mathrm{D}$ & $\mathrm{A}$ & $\mathrm{TA}$ & Ítem & TD & $\mathrm{D}$ & $\mathrm{A}$ & $\mathrm{TA}$ \\
\hline 1 & -2 & -1 & 1 & 2 & 1 & -2 & -1 & 1 & 2 \\
\hline 2 & 2 & 1 & -1 & -2 & 2 & 2 & 1 & -1 & -2 \\
\hline 3 & 2 & 1 & -1 & -2 & 3 & 2 & 1 & -1 & -2 \\
\hline 4 & -2 & -1 & 1 & 2 & 4 & -2 & -1 & 1 & 2 \\
\hline 5 & 2 & 1 & -1 & -2 & 5 & 2 & 1 & -1 & -2 \\
\hline 6 & 2 & 1 & -1 & -2 & 6 & 2 & 1 & -1 & -2 \\
\hline 7 & -2 & -1 & 1 & 2 & 7 & -2 & -1 & 1 & 2 \\
\hline 8 & -2 & -1 & 1 & 2 & 8 & -2 & -1 & 1 & 2 \\
\hline 9 & -2 & -1 & 1 & 2 & 9 & -2 & -1 & 1 & 2 \\
\hline 10 & -2 & -1 & 1 & 2 & 10 & -2 & -1 & 1 & 2 \\
\hline
\end{tabular}

Fuente: elaboración propia.

* Los ítems evaluados con no sabe/no contesta son otorgados un valor de cero puntos

Como resultado de la aplicación de cada cuestionario se obtiene una puntuación de la suma total de las calificaciones asignadas a las respuestas, cuyo significado representa, respectivamente, el grado de importancia otorgado al desarrollo del pensamiento crítico del alumnado en el centro desde cada una de las posiciones estudiadas, los cuales pueden obtener valores entre -20 (el sujeto considera el pensamiento crítico del alumnado no es nada promovido en el centro) y 20 (opina que se promueve mucho el pensamiento crítico del alumnado).

Además, para poder tratar de una forma más visual los datos recabados de las respuestas a los distintos ítems del cuestionario, se emplean grados de acuerdo y desacuerdo. Dichos grados se calculan para cada ítem, ponderando el número total de respuestas en cada rango de la escala coherentemente con la codificación establecida en la Tabla 3, de la siguiente manera:

$$
\begin{gathered}
\text { Grado de acuerdo }(\%)=\frac{N^{\mathrm{o}} A+\left(2 \times N^{\circ} T A\right)}{\left(2 \times N^{\circ} T D\right)+N^{\circ} D+N^{\circ} A+\left(2 \times N^{\circ} T A\right)} * 100 \\
\text { Grado de desacuerdo }(\%)=\frac{N^{\circ} D+\left(2 \times N^{\circ} T D\right)}{\left(2 \times N^{\circ} T D\right)+N^{\circ} D+N^{\circ} A+\left(2 \times N^{\circ} T A\right)} * 100
\end{gathered}
$$

De esta forma, se obtienen dos porcentajes que permiten representar la respuesta recibida de una forma más manejable, o, lo que es lo mismo, facilitan el análisis del punto de vista de los encuestados.

\subsubsection{Datos cualitativos}

En el tratamiento de los datos cualitativos se efectúa un análisis de contenidos, cuyo objetivo es presentar un resumen de la narrativa dominante en el discurso evaluado, recopilando, resaltando y analizando, simultáneamente, los fragmentos de los discursos obtenidos durante la recogida de información. Además, se presentan y estudian los puntos de vista divergentes de la narrativa general, persiguiendo dotar al análisis de una perspectiva lo más amplia posible.

\section{Resultados}

\subsection{Análisis de la posición docente}

De los 60 docentes que conforman la muestra respondieron 25, suponiendo una participación del $42 \%$. La participación por género presentó porcentajes similares a la participación global, por lo que 
no hubo diferencias notables en este aspecto. La respuesta por departamentos fue diversa, participando en el estudio docentes de diez departamentos didácticos diferentes de los quince que conforman el centro.

En la Figura 2, se presentan los resultados obtenidos de la parte cuantitativa del cuestionario «Percepción docente sobre el desarrollo del pensamiento crítico del alumnado en ESO y Bachillerato».

Figura 2.

Resultados cuantitativos del cuestionario «Percepción docente sobre el desarrollo del pensamiento crítico del alumnado en ESO y Bachillerato»

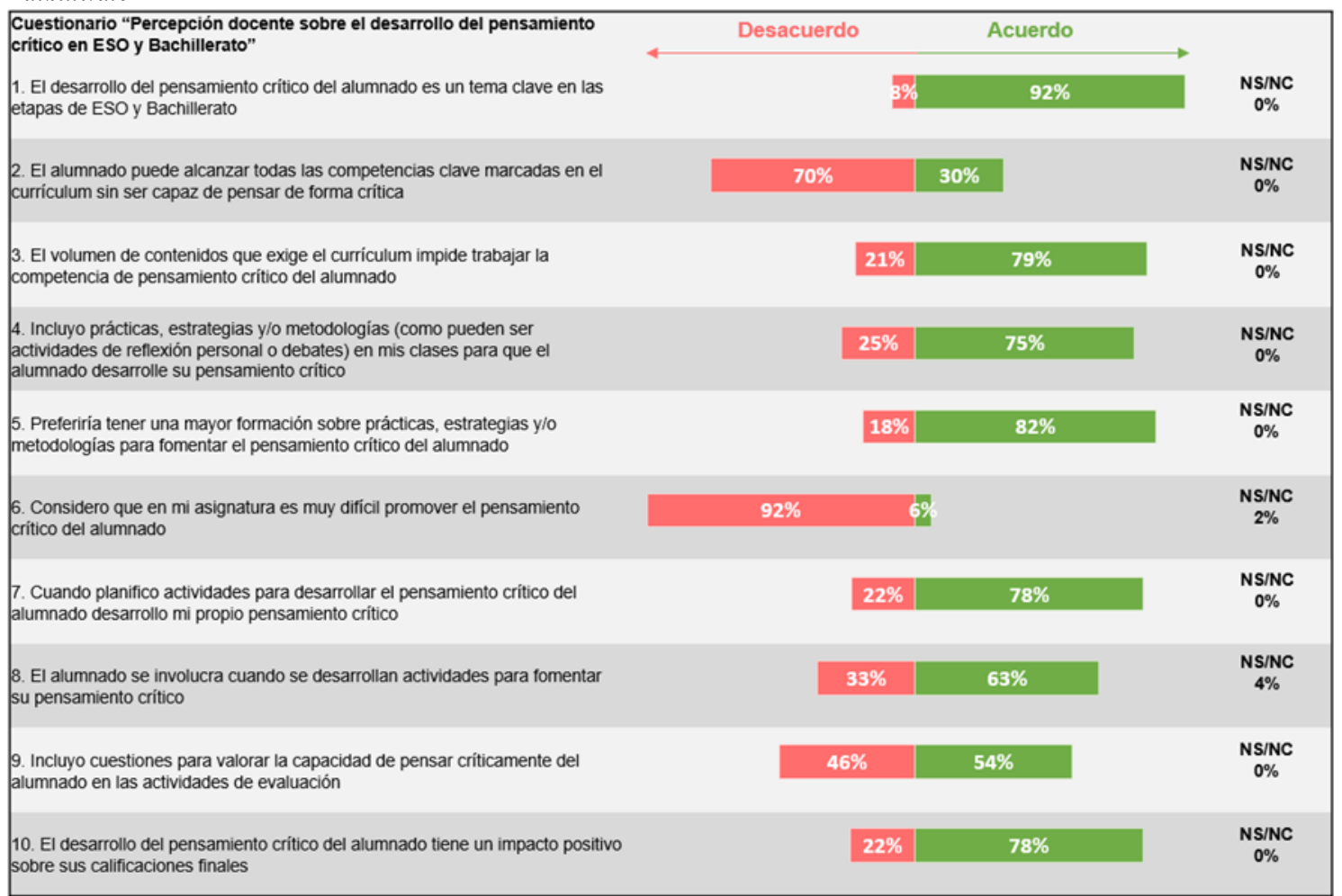

Fuente: elaboración propia

Como puede observarse en la Figura 2, los docentes del centro que responden al cuestionario, muestran un grado de acuerdo significativo $(92 \%)$ sobre el hecho de que el desarrollo del pensamiento crítico del alumnado constituye un aspecto importante en ESO y Bachillerato. La mayoría del profesorado del centro $(70 \%)$ no cree que el alumnado sea capaz de alcanzar el nivel de desarrollo de las competencias clave marcadas por el currículum sin pensar de una forma crítica. Sin embargo, ven el elevado volumen de contenidos exigido por el currículum como una barrera a la hora de promover el pensamiento crítico del alumnado (acuerdo del $75 \%$ ).

Aun así, y pese a que la mayoría $(82 \%)$ preferiría poseer mayor formación en prácticas, estrategias y metodologías para promover el pensamiento crítico del alumnado, un gran porcentaje del profesorado del centro $(75 \%)$ afirma incluir prácticas, estrategias y metodologías en sus sesiones para fomentar este aspecto. Casi la totalidad del profesorado $(92 \%)$ considera que en su asignatura no es difícil promover el pensamiento crítico del alumnado. Además, los docentes están de acuerdo $(78 \%)$ con la afirmación de que planificar actividades para desarrollar el pensamiento crítico del alumnado les ayuda a fomentar su propio pensamiento crítico y, aseveran también, que el grado de involucración de los estudiantes cuando se desarrollan actividades para potenciar este aspecto es, por lo general, bueno (acuerdo del 63\%).

Existe una mayor variedad de respuestas en cuanto a la inclusión de cuestiones para valorar el pensamiento crítico del alumnado en las actividades de evaluación (acuerdo del 54\% y desacuerdo del $46 \%$ ). En lo respectivo al impacto del pensamiento crítico del alumnado sobre sus calificaciones, los docentes señalan (acuerdo del 78\%), que el desarrollo de esta capacidad significa la obtención de mejores calificaciones.

En cuanto a la valoración global del cuestionario, se obtuvo que la media de la variable PC-Docente, es decir, el grado en que los docentes consideran que el pensamiento crítico del alumnado es 
promovido del centro, fue de $\overline{\mathrm{V} 1}=3.68$, d.t. $=4.28$, siendo ligeramente superior al valor promedio de la escala (0).

Ante la pregunta que solicita expresar la concepción sobre el pensamiento crítico, las respuestas más destacadas se presentan en la Tabla 4.

Tabla 4.

Conceptualización del pensamiento crítico por parte del profesorado

\begin{tabular}{|c|c|}
\hline Conceptualización & Participante \\
\hline $\begin{array}{l}\text { «La capacidad de cuestionar tanto lo que nos ha sido transmitido } \\
\text { como nuestro propio pensamiento. Se puede llevar a cabo a través } \\
\text { de la observación, interiorización y de la reflexión, tratando de que } \\
\text { este proceso introspectivo lleve a algún tipo de conclusión.» }\end{array}$ & $\begin{array}{l}\text { Docente del Dpto. de Tecnología } \\
\text { (Género femenino, 0-5 años expe- } \\
\text { riencia docente) }\end{array}$ \\
\hline $\begin{array}{l}\text { «Capacidad para seleccionar y utilizar la información comprendién- } \\
\text { dola, cuestionándola y sirviéndose de ella para formar ideas propias } \\
\text { capaces de mejorar la vida personal y social. Incluye la capacidad de } \\
\text { análisis y cuestionamiento de las propias ideas.» }\end{array}$ & $\begin{array}{l}\text { Docente del Dpto. de Filosofía (Gé- } \\
\text { nero femenino, }+25 \text { años experiencia } \\
\text { docente) }\end{array}$ \\
\hline $\begin{array}{l}\text { «Capacidad de decidir, opinar y justificar algo bajo argumentos pro- } \\
\text { pios, sin estar influenciado por nada ni nadie externo.» }\end{array}$ & $\begin{array}{l}\text { Docente del Dpto. de Orientación } \\
\text { (Género femenino, 15-25 años expe- } \\
\text { riencia docente) }\end{array}$ \\
\hline
\end{tabular}

Fuente: elaboración propia

En resumen, el grueso de las respuestas se fundamenta sobre tres pilares o capacidades que los docentes consideran que son fundamentales del pensamiento crítico: 1. cuestionar la información recibida y reflexionar sobre ella; 2 . crear una visión propia y personal; 3. analizar e interpretar la información.

Entre las prácticas, estrategias o metodologías que los docentes utilizan en sus clases para fomentar el pensamiento crítico del alumnado, destacan las respuestas recogidas en la Tabla 5.

Tabla 5.

Prácticas, estrategias o metodologías que los docentes utilizan para fomentar el pensamiento crítico del alumnado (perspectiva docente)

\begin{tabular}{l}
\hline Prácticas, estrategias o metodologías \\
«Mediante preguntas sobre temas tratados en clase y trabajos de in- \\
vestigación donde tienen que poner sus opiniones.»
\end{tabular}

vestigación donde tienen que poner sus opiniones.»

«Análisis y comentario de textos: comprende tanto el análisis del texto para su comprensión como el comentario crítico (plantear preguntas al texto, establecer relaciones con otros conocimientos adquiridos, buscar ideas y argumentos contradictorios, etc.)»

«Análisis de objetos, preguntas indirectas, ejercicios de cálculo no memorístico, desarrollo de proyectos, forzar la creatividad, el pensamiento lateral (siempre existe más de una forma de ver o hacer las cosas), no dar las soluciones mascadas, etc..»

«A través de textos, preguntas guiadas para llegar a la meta, redactar opiniones personales sobre lecturas relacionándolos con sus propias experiencias y conocimientos. Saber escuchar también es imporParticipante

Docente del Dpto. de Tecnología

(Género femenino, 15-25 años experiencia docente)

Docente del Dpto. de Filosofía (Género femenino, +25 años experiencia docente)

Docente del Dpto. de Tecnología (Género masculino, 15-25 años experiencia docente)

Docente del Dpto. de Lengua (Género femenino, $0-5$ años experiencia docente)

tante.»

Fuente: elaboración propia

Las prácticas, estrategias y metodologías que toman protagonismo, pueden agruparse en: 1. preguntas para debatir en el aula sobre temas tratados en clase; 2 . solicitar producciones escritas que reflejen la opinión personal; 3. análisis y comentario de textos. Además, dos docentes señalan el aprendizaje cooperativo como metodología que emplean para desarrollar el pensamiento crítico del 
alumnado. Respecto a la evaluación de dichas prácticas, estrategias y metodologías, ningún docente respondió a esta cuestión.

\subsection{Análisis de la posición del alumnado}

En el caso del alumnado respondieron al cuestionario 43 de los 45 encuestados. En la Figura 3, se presentan los resultados obtenidos de la parte cuantitativa del cuestionario «Percepción del alumnado sobre el desarrollo del pensamiento crítico en ESO y Bachillerato».

Figura 3.

Resultados cuantitativos del cuestionario «Percepción del alumnado sobre el desarrollo del pensamiento crítico en ESO y Bachillerato»)

\begin{tabular}{|c|c|c|c|}
\hline $\begin{array}{l}\text { Cuestionario "Percepción del alumnado sobre el desarrollo del } \\
\text { pensamiento critico en ESO y Bachillerato" }\end{array}$ & Desacuerdo & Acuerdo & \\
\hline $\begin{array}{l}\text { 1. El desarrollo de mi capacidad para pensar de forma crítica es un tema clave } \\
\text { en las etapas de ESO y Bachillerato }\end{array}$ & $25 \%$ & $72 \%$ & $\underset{3 \%}{\mathrm{NS} / \mathrm{NC}}$ \\
\hline $\begin{array}{l}\text { 2. Puedo alcanzar los objetivos educativos de cada curso académico sin ser } \\
\text { capaz de pensar de una forma crítica }\end{array}$ & $47 \%$ & $43 \%$ & $\begin{array}{c}\text { NS/NC } \\
10 \%\end{array}$ \\
\hline $\begin{array}{l}\text { 3. La gran cantidad de contenidos que tengo que estudiar me impide } \\
\text { detenerme a pensar sobre ellos de forma crítica }\end{array}$ & $41 \%$ & $53 \%$ & $\begin{array}{c}\text { NS/NC } \\
5 \%\end{array}$ \\
\hline $\begin{array}{l}\text { 4. Mis profesores/as incluyen prácticas, estrategias y/o metodologias (como } \\
\text { pueden ser actividades de reflexión personal o debates) en sus clases para } \\
\text { que los alumnos/as desarrollemos la capacidad de pensar de forma crítica }\end{array}$ & $57 \%$ & $36 \%$ & $\begin{array}{c}\text { NS/NC } \\
7 \%\end{array}$ \\
\hline $\begin{array}{l}\text { 5. Me gustaría que los profesores/as dedicaran más tiempo a fomentar las } \\
\text { capacidades de los alumnos/as para pensar de forma crítica }\end{array}$ & $22 \%$ & $69 \%$ & $\begin{array}{c}\text { NS/NC } \\
9 \%\end{array}$ \\
\hline $\begin{array}{l}\text { 6. En varias asignaturas es muy dificil para los profesores/as diseñar } \\
\text { actividades para que los alumnos/as desarrollemos nuestra capacidad para } \\
\text { pensar de forma crítica }\end{array}$ & $34 \%$ & $64 \%$ & $\begin{array}{l}\text { NS/NC } \\
2 \%\end{array}$ \\
\hline $\begin{array}{l}\text { 7. Considero útiles las actividades para desarrollar mi capacidad para pensar } \\
\text { de forma crítica (como pueden ser actividades de reflexión personal o debates) }\end{array}$ & $15 \%$ & $83 \%$ & $\begin{array}{c}\text { NS/NC } \\
2 \%\end{array}$ \\
\hline $\begin{array}{l}\text { 8. Los alumnos/as nos involucramos cuando se desarrollan actividades para } \\
\text { fomentar nuestro pensamiento crítico }\end{array}$ & $24 \%$ & $69 \%$ & $\begin{array}{c}\mathrm{NS} / \mathrm{NC} \\
7 \%\end{array}$ \\
\hline $\begin{array}{l}\text { 9. Mis profesores/as incluyen actividades para valorar la capacidad de pensar } \\
\text { criticamente de los alumnos/as en las actividades de evaluación }\end{array}$ & $58 \%$ & $31 \%$ & $\begin{array}{c}\text { NS/NC } \\
12 \%\end{array}$ \\
\hline $\begin{array}{l}\text { 10. El desarrollo de mi capacidad para pensar de forma critica tiene un impacto } \\
\text { positivo sobre mis calificaciones finales }\end{array}$ & $23 \%$ & $76 \%$ & $\begin{array}{c}\text { NS/NC } \\
2 \%\end{array}$ \\
\hline
\end{tabular}

Fuente: elaboración propia

Como puede observarse en la Figura 3, la mayor parte del alumnado encuestado está de acuerdo $(72 \%)$ con que el desarrollo de su pensamiento crítico constituye un aspecto importante las etapas de ESO y Bachillerato. Cuando son cuestionados sobre si creen que son capaces de alcanzar los objetivos educativos de cada curso académico sin pensar de forma crítica, existe división de opiniones ( $47 \%$ desacuerdo; $43 \%$ acuerdo; $10 \%$ NS/NC). En cuanto al impacto del volumen de contenidos exigido por el currículum en su capacidad para reflexionar críticamente sobre ellos, la balanza se inclina ligeramente (acuerdo del 53\%) hacia la opinión de que el volumen de contenidos impide dicha reflexión por ser demasiado elevado.

Respecto a las prácticas, estrategias y metodologías para fomentar el pensamiento crítico del alumnado, hay un porcentaje significativo del alumnado $(57 \%)$ que señala que sus docentes no las incorporan en sus sesiones. Por otra parte, la mayor parte del alumnado (acuerdo del 64\%) cree que en algunas asignaturas es muy difícil para los docentes diseñar actividades para promover el pensamiento crítico del alumnado. Un porcentaje muy importante del alumnado afirma considerar útiles las actividades para desarrollar su pensamiento crítico $(83 \%)$ y, además, indican que les gustaría que sus docentes dedicasen más tiempo a fomentar este aspecto (acuerdo del 69\%), señalando también que se involucran de manera positiva en este tipo de actividades (acuerdo del 69\%).

En lo relativo a la evaluación, la mayor parte del alumnado (58\%) considera que sus docentes no incluyen cuestiones en sus pruebas para valorar su pensamiento crítico. El alumnado indica que el 
desarrollo de su pensamiento crítico tiene un impacto positivo sobre sus calificaciones (acuerdo del $76 \%)$.

En cuanto a la valoración global de la parte cuantitativa del cuestionario, se obtuvo que la media de la variable PC-Alumnado, es decir, el grado en que los estudiantes consideran que su pensamiento crítico es promovido del centro, fue de $\mathrm{M}=1.07$, d.t. $=4.25$, siendo, de igual manera que en el caso de los docentes, ligeramente superior al valor promedio de la escala.

Cuando el alumnado es preguntado sobre su concepción sobre el pensamiento crítico, entre sus respuestas destacan las citadas en la Tabla 6.

Tabla 6.

Conceptualización del pensamiento crítico por parte del alumnado

\begin{tabular}{ll}
\hline Conceptualización & Participante \\
\hline «Pensar y analizar situaciones desde distintos puntos de vista y ser capaz de & Alumna de $3^{\circ} \mathrm{ESO}$ \\
formar una idea individual.» & \\
«Analizar, entender y evaluar la manera en que se organizan los conocimientos & Alumna de $4^{\circ} \mathrm{ESO}$ \\
que se pretenden interpretar.» & \\
«Un pensamiento personal que te ayuda a entender la información recibida.» & Alumno de $1^{\circ}$ Bachillerato \\
«La capacidad de pensar por ti mismo y, a partir de datos objetivos, elaborar & Alumna de $2^{\circ}$ Bachillerato \\
una conclusión propia para construir tus conocimientos.» & \\
\hline
\end{tabular}

Fuente: elaboración propia

Se observan como aspectos que el alumnado destaca sobre el pensamiento crítico: 1. capacidad de análisis y reflexión; 2. construcción de un pensamiento personal; 3. comprensión de la información recibida.

En relación a las prácticas, estrategias y metodologías que el profesorado incluye en sus sesiones para fomentar el pensamiento crítico del alumnado, destacan las respuestas por parte del alumnado recogidas en la Tabla 7.

Tabla 7.

Prácticas, estrategias o metodologías que los docentes utilizan para fomentar el pensamiento crítico del alumnado (perspectiva del alumnado)

\begin{tabular}{ll}
\hline Prácticas, estrategias o metodologías & Participante \\
\hline «De vez en cuanto abrimos pequeños debates en clase» & Alumna de $3^{\circ} \mathrm{ESO}$ \\
«Salidas del centro, experimentos de laboratorio, aprendizaje cooperativo» & Alumna de $4^{\circ} \mathrm{ESO}$ \\
«Preguntar nuestra opinión respecto a un tema y trabajos de investigación» & Alumno de $1^{\circ}$ Bachillerato \\
«Trabajos en grupo y elaboración de comentarios críticos» & Alumna de $2^{\circ}$ Bachillerato \\
\hline
\end{tabular}

Fuente: elaboración propia

Las prácticas, estrategias o metodologías que el alumnado indica que el profesorado utiliza para fomentar su pensamiento crítico se dividen en 6 grupos: 1. debates en el aula; 2. aprendizaje cooperativo; 3. preguntas de opinión; 4. trabajos de investigación; 5 . comentarios críticos de textos; 6 . actividades extraescolares. Respecto a la evaluación de este tipo de actividades, se ha obtenido un bajo volumen de respuesta. Una alumna de $2^{\circ}$ Bachillerato señala que «los debates no se evalúan y los comentarios de texto sí que cuentan parte de la nota para ciertas asignaturas», aunque no indica la metodología empleada, por lo que no es posible desarrollar más sobre este aspecto.

Por otra parte, se ha recibido un volumen importante de respuestas (más del 50\% del alumnado cada grupo) en las que los estudiantes indican que sus docentes no incluyen prácticas, estrategias o metodologías para fomentar su pensamiento crítico, formulando respuestas como: «no las usan, hacen la media de trabajos, exámenes y comportamiento» (alumna de $3^{\circ} \mathrm{ESO}$ ); «el profesorado no trata de fomentar nuestra capacidad de pensar críticamente» (alumna de $4^{\circ} \mathrm{ESO}$ ); «no utilizan ninguna, como mucho un debate al año» (alumno de $1^{\circ}$ Bachillerato); «no hemos desarrollado este tipo de actividades o, al menos, yo no las he percibido» (alumno de $2^{\circ}$ Bachillerato).

\section{Discusión y conclusiones}

La investigación permite determinar que, tanto el profesorado como el alumnado del centro analizado, indican que la promoción del pensamiento crítico del alumnado constituye un aspecto clave en 
las etapas de ESO y Bachillerato, lo cual se encuentra en la línea de las palabras de Elder y Paul (2003) y Causado, Santos y Calderón (2015). Sin embargo, las medias obtenidas de la valoración numérica de la parte cuantitativa de los cuestionarios, aun siendo positivas, se encuentran muy próximas a la neutralidad, no siendo posible afirmar que ninguno de los dos colectivos considere que el pensamiento crítico del alumnado es promovido en el centro.

En la línea de este hecho, el profesorado y el alumnado del centro reclaman que se brinde mayor atención hacia el desarrollo del pensamiento crítico del alumnado en ESO y Bachillerato: el alumnado pide que los docentes dediquen más tiempo a fomentar este aspecto y, los docentes, solicitan actividades de formación para poder hacerlo de manera apropiada, correspondiéndose con lo reportado por Kowalczyk, Hackworth y Case-Smith (2012).

Ambos colectivos perciben la utilidad de las actividades para desarrollar el pensamiento crítico del alumnado: los docentes están de acuerdo con que este tipo de actividades les ayuda a fomentar su propio pensamiento crítico y el alumnado señala que las considera útiles, por lo que todos salen beneficiados de su aplicación. Además, incluir prácticas, estrategias y metodologías eficaces para promover el pensamiento crítico del alumnado, de acuerdo a la opinión de los docentes y discentes del centro, resultaría en la obtención de mejores calificaciones por parte de los últimos, coincidiendo con las observaciones de Innabi y El Sheikh (2006) y de Chee y Kin (2009).

$\mathrm{El}$ aspecto menos robusto, y que requiere más atención para fortalecer la promoción del pensamiento crítico del alumnado del centro, en la línea de lo expuesto por Dumitru (2018), se identifica en las fases de evaluación: no hay consenso sobre si los docentes actualmente incluyen cuestiones para valorar este aspecto en las actividades de evaluación.

En lo que respecta a la conceptualización del pensamiento crítico, las respuestas de los participantes en la investigación, indican una ausencia de consenso en cuanto al significado de este término, lo cual se corresponde con las ideas expuestas por varios autores (Díaz, 2001; Lipman, 1997; OssaCornejo, Palma-Luengo, Lagos-San Martín, Quintana-Abello \& Díaz-Larenas, 2017). Pese a esto, dichas respuestas mantienen coherencia con la idea fundamental de que el pensamiento crítico es un pensamiento racional orientado a revisar y evaluar ideas y argumentos (Zelaieta \& Camino, 2018). Al contrario de lo expresado por Alazzi (2008), Aliakbari \& Sadeghdaghighi (2013), Chee \& Kin (2009) y Innabi y El Sheikh (2006), los docentes no cometieron errores a la hora de conceptualizar el pensamiento crítico.

Se observó dificultad por parte de los participantes para identificar prácticas, estrategias y metodologías que indirectamente favorecen el desarrollo del pensamiento crítico del alumnado, lo que indica que la concepción que ambos colectivos poseen sobre el pensamiento crítico es susceptible de mejora. Realizar actividades de formación para los docentes e incluir actividades en sesiones de tutoría con el objetivo de educar a los estudiantes sobre el concepto de pensamiento crítico y concienciarles sobre la importancia de su desarrollo podría constituir una posible vía de acción, con el objetivo de reforzar la forma en el que el pensamiento crítico del alumnado se fomenta en el centro educativo analizado.

Sobre las limitaciones de la investigación, destaca especialmente el tamaño reducido de la muestra, que compromete la representatividad de los resultados obtenidos. De cara a futuras investigaciones, sería interesante efectuar un estudio que proporcione resultados representativos en el contexto de un IES. Además, podría darse continuidad a este trabajo contrastando si los resultados obtenidos pueden extrapolarse a otros IES de la provincia en la que se contextualiza la investigación o, incluso, a nivel nacional, con el objetivo de crear una visión más completa sobre la perspectiva de docentes y estudiantes de ESO y Bachillerato en relación a la promoción del pensamiento crítico de los últimos, y que es un aspecto clave en el contexto actual.

\section{Referencias}

Alazzi, K. (2008). Teachers' perceptions of critical thinking: A study of Jordanian secondary school social studies teachers. The social studies, 99(6), 243-248. doi: 10.3200/TSSS.99.6.243-248

Albert, M.J. (2007). La investigación educativa: claves teóricas. Madrid: McGraw-Hill.

Albertos, D., \& De la Herrán, A. (2018). Desarrollo del pensamiento crítico en estudiantes de Educación Secundaria: diseño, aplicación y evaluación de un programa educativo. Profesorado, Revista de Currículum y Formación del Profesorado, 22(4), 269-285. doi: 10.30827/profesorado.v22i4.8416

Aliakbari, M., \& Sadeghdaghighi, A. (2013). Teachers' perception of the barriers to critical thinking. Procedia-Social and Behavioral Sciences, 70, 1-5. doi: 10.1016/j.sbspro.2013.01.031 
Allamnakhrah, A. (2013). Learning Critical Thinking in Saudi Arabia: Student Perceptions of Secondary Pre-Service Teacher Education Programs. Journal of Education and learning, 2(1), 197-210.

Bisquerra, R. (2004). Metodología de la investigación educativa. Madrid: La muralla.

Briceño, M. \& Romero, R. (2012). Aplicación del método Delphi para la validación de los instrumentos de evaluación del libro electrónico multimedia "Andrés quiere una mascota". Anales de la Universidad Metropolitana, 12(1), 37-67. Recuperado de: https://idus.us.es/xmlui/handle/11441/39721

Cárdenas, C., Farías, G. M., \& Méndez, G. (2017). ¿Existe relación entre la gestión administrativa y la innovación educativa?: Un estudio de caso en educación superior. REICE. Revista Iberoamericana sobre Calidad, Eficacia y Cambio en Educación, 15(1), 19-35. doi: 10.15366/reice2017.15.1.002

Causado, R., Santos, B., \& Calderón, S. (2015). Desarrollo del pensamiento crítico en el área de ciencias naturales en una escuela de secundaria. Revista de la Facultad de Ciencias, 4(2), 17-42. doi: $10.15446 /$ rev.fac.cienc.v4n2.51437

Chee, S., \& Kin, P. (2009). Teacher perceptions of critical thinking among students and its influence on higher education. International Journal of teaching and learning in Higher Education, 20(2), 198-206.

Díaz, F. (2001). Habilidades de pensamiento crítico sobre contenidos históricos en alumnos de bachillerato. Revista Mexicana de Investigación Educativa, 6(13), 525-554. Recuperado de: http://www.redalyc.org/articulo.oa?id=14001308

Dumitru, D. (2018). Teachers' Thoughts About How Critical Thinking Is a Part of Their Classes. En International Conference on Technology and Innovation in Learning, Teaching and Education (pp. 203-209). Springer, Cham.

Dumitru, D., Bigu, D., Elen, J., Jiang, L., Railienè, A., Penkauskienè, D., ... \& McNally, C. (2018). A European review on critical thinking educational practices in higher education institutions. Vila Real: UTAD.

Elder, L., \& Paul, R. (2003). Los fundamentos del pensamiento analitico. California: The Foundation for Critical Thinking.

Innabi, H., \& El Sheikh, O. (2007). The change in mathematics teachers' perceptions of critical thinking after 15 years of educational reform in Jordan. Educational Studies in Mathematics, 64(1), 45-68. doi: 10.1007/s10649-005-9017-x

Kowalczyk, N., Hackworth, R., \& Case-Smith, J. (2012). Perceptions of the use of critical thinking teaching methods. Radiologic technology, 83(3), 226-236.

Lima, M., Âvila, A., \& López, C. (2015). Influencia de los Antecedentes Académicos para el Ingreso en una Universidad Pública Mexicana: Un Estudio de Caso. REICE. Revista Iberoamericana sobre Calidad, Eficacia y Cambio en Educación, 13(3), 107-119. doi: 10.15366/reice2017.15.1.002

Lipman, M. (1997). Pensamiento complejo y educación. Madrid: Ediciones de la Torre.

Ley Orgánica 2/2006, de 3 de mayo, de Educación (LOE). Boletín Oficial del Estado, núm. 295, de 4 de mayo de 2006, pp. 17158-17207.

Ley Orgánica 8/2013, de 9 de diciembre, para la mejora de la calidad educativa (LOMCE). Boletín Oficial del Estado, núm. 295, de 10 de diciembre de 2013, pp. 97858-97921.

Lozano, J. M., \& Vieites, A. (2017). Gestión de la Calidad y Diseño Específico de los Procesos de Admisión en el Sistema Universitario Español: Estudio de Caso en una Universidad Privada. REICE. Revista Iberoamericana sobre Calidad, Eficacia y Cambio en Educación, 15(1), 87-106. doi: 10.15366/reice2017.15.1.006

Martínez, P., Ballester, J., \& Ibarra, N. (2018). Estudio cuasiexperimental para el análisis del pensamiento crítico en secundaria. Revista Electrónica de Investigación Educativa, 20(4), 123-132. doi: 10.24320/redie.2018.20. 4.1705

Ossa-Cornejo, C.J., Palma-Luengo, M.R., Lagos-San Martín, N.G., Quintana-Abello, I.M., y DíazLarenas, C.H. (2017). Análisis de instrumentos de medición del pensamiento crítico. Ciencias Psicológicas, 11(1), 19-28. doi: 10.22235/cp.v11i2.1343

Salcines Talledo, I., \& González Fernández, N. (2015). Diseño y Validación del Cuestionario "Smartphone y Universidad. Visión del Profesorado" (SUOL). Revista Complutense De Educación, 27(2), 603-632. doi: 10.5209/rev_RCED.2016.v27.n2.46912

Tapper, J. (2004). Student perceptions of how critical thinking is embedded in a degree program. Higher education research \& Development, 23(2), 199-222. doi: 10.1080/0729436042000206663

Úbeda-Colomer, J., \& Molina, J.P. (2016). El blog como herramienta didáctica en educación física: la percepción del alumnado. Apunts. Educación Física y Deportes, 126, 37-45. doi: 10.5672/apunts.20140983.es.(2016/4).126.04 
Yazan, B. (2015). Three approaches to case study methods in education: Yin, Merriam, and Stake. The qualitative report, 20(2), 134-152. Recuperado de: https://nsuworks.nova.edu/tqr/vol20/iss2/12

Zelaieta, E., \& Camino, I. (2018). El desarrollo del pensamiento crítico en la formación inicial del profesorado: análisis de una estrategia pedagógica desde la visión del alumnado. Revista de currículum y formación del profesorado, 22(1), 197-214. Recuperado de: https://recyt.fecyt.es/index.php/profesorado/article/view/63641 